(OR 1.77; CI 95\% 1.05, 2.99), edad materna de por lo menos 35 años (OR 1.50; IC 95\% 1.14, 2.01), madre soltera (OR 1.50; IC 95\% 1.13, 1.98), fumar cigarrillo (OR 1.40; IC 95\% 1.00, 1.97 para 10 a 19 cigarrillos por día y OR 1.13 , IC $95 \% 0.81,1.59$ para al menos 20 cigarrillos al día) y sexo fetal masculino (OR 1.38; IC $95 \% 1.12,1.70$ ). La remoción de los bebés muy pequeños para la edad gestacional produjo poco cambio en la magnitud de las otras asociaciones.

Conclusiones: El retardo de crecimiento fetal severo, ruptura prolongada de membranas, corioamnionitis, hipertensión (antes de la gestación o inducida por la gestación), fumar cigarrillo, edad materna avanzada, estado marital soltera y el sexo fetal masculino son determinantes etiológicas significativas del abrutio placentae. Las determinantes diferentes del retardo severo del crecimiento fetal parecen operar muy independientemente de sus efectos sobre el crecimiento fetal.

\section{Concentraciones de hCG en el fluído vaginal para la detección de ruptura prematura de membranas}

Takanobu Anai, MD, Yuichirou Tanaka, MD, Yoshiko Hirota, MD, and Isao Miyakawa, MD

\section{From the Department of Obstetrics and Gynecology, Oita Medical University, Oita, Japan}

\section{Obstet Gynecol 1997; 89: 261-264.}

Objetivo: Determinar si la medición de los niveles de hCG en el fluido vaginal es útil para el diagnóstico de la ruptura prematura de membranas (RPM).

Métodos: Se midieron las concentraciones de hCG después de irrigar el fondo de saco vaginal posterior con $3 \mathrm{~mL}$ de solución salina estéril y obtener lavados vaginales. Se analizaron las muestras de 188 mujeres gestantes normales, 42,61 y 85 durante el primero, segundo y tercer trimestres, respectivamente. Los niveles de hCG se compararon con aquellos de 24 mujeres con RPM confirmada.

Resultados: La medida e intervalo de confianza del $95 \%$ de los niveles de hCG en fluido vaginal de mujeres embarazadas normales fueron $37.9(1.9,725.6), 9.5(0.8,95.8)$ y 6.3 $(0.6,62.2) \mathrm{mUI} / \mathrm{mL}$ durante el primero, segundo y tercer trimestres, respectivamente. Los de las mujeres con RPM fueron $420.6(216.3,918.3) \mathrm{mUI} / \mathrm{mL}$. Para el segundo trimestre la sensibilidad fue del $100 \%$, especificidad del $91.8 \%$, valor predictivo positivo del $82.8 \%$, valor predictivo negativo del $100 \%$ y exactitud del $94.1 \%$; y para el tercer trimestre la sensibilidad fue del $100 \%$, especificidad del $96.5 \%$, valor predictivo positivo del $88.9 \%$, valor predictivo negativo del $100 \%$ y exactitud del $97.2 \%$, utilizando un valor límite de $50 \mathrm{mUI} / \mathrm{mL}$.

Conclusión: La concentración de hCG en el fluido vaginal es un marcador útil de RPM durante el segundo y el tercer trimestres.

\section{Efectos a corto plazo de la testosterona tópica en el liquen escleroso vulvar}

Elmar A. Joura, MD, Harald Zeisler, MD, Dagmar Bancher-Todesca, MD, Michael O. Sator, MD, Barbara Schneider, PhD, and Gerald Gitsch, MD
From the Department of Gynecology and Obstetrics, Division of Gynecological Endocrinology and Sterility, and Institute of Medical Statistics, University of Vienna Medical School-AKH, Vienna, Austria

$$
\text { Obstet Gynecol 1997; 89: 297-299. }
$$

Objetivo: Evaluar los efectos sistémicos y terapéuticos del tratamiento con testosterona tópica del liquen escleroso vulvar.

Métodos: Este estudio clínico prospectivo incluyó 10 mujeres post-menopáusicas con liquen escleroso vulvar. Se administró propionato de testosterona $(0.04 \mathrm{~g}$ al día) en forma tópica por 4 semanas. Se determinaron las concentraciones séricas de andrógenos (testosterona, androstendiona y dehidroepiandrosterona) antes y después de 4 semanas de tratamiento, y la vulvodinia se evaluó por una escala visual análoga horizontal.

Resultados: Las concentraciones séricas de testosterona total aumentaron en todas las pacientes $(p<.01)$ y sobrepasaron el rango normal en ocho de diez mujeres. La vulvodinia mejoró en 9 de 10 mujeres (test $t$ pareado: $p$ $<.01$ ). Cuadro de diez mujeres presentaron signos clínicos de hiperandrogenismo (aumento del tamaño del clítoris, alteraciones de la voz, aumento de la líbido) después de 4 semanas de tratamiento. La única paciente sin mejoría subjetiva tenía niveles basales elevados de andrógenos y presentaba signos clínicos de hiperandrogenismo antes de la terapia.

Conclusión: La testosterona tópica es efectiva en la mujer normoandrogénica con liquen escleroso. El estado androgénico debe ser evaluado antes del tratamiento, y la dosis debe ser individualizada para evitar la virilización y los efectos metabólicos colaterales. Se recomiendan controles clínicos y un seguimiento con evaluación de los niveles séricos de testosterona, debido a que hay un marcado efecto sistémico. Se deben incluir otros esteroides en las decisiones terapéuticas.

\section{Cirugía reconstructiva vaginal versus abdominal para el tratamiento de los defectos del soporte pélvico: un estudio prospectivo aleatorizado con evaluación de resultados a largo plazo}

J. Thomas Benson, MD, ${ }^{a}$ Vincent Lucente, MD, ${ }^{b}$ and Elizabeth McClellan, RN, BSN ${ }^{c}$

From the Department of Obstetrics and Gynecology, Methodist Hospital Medical Center, ${ }^{a}$ the Department of Obstetrics and Gynecology, Lehigh Valley Hospital, ${ }^{b}$ and Urogynecology Associates. ${ }^{c}$

Am J Obstet Gynecol 1996; 175: 1418-1422.

Objetivo: Determinar si la aproximación vaginal o la abdominal es más efectiva para corregir el prolapso uterovaginal.

Diseño del estudio: Se aleatorizaron para intervención quirúrgica vaginal versus abdominal, a 88 mujeres con prolapso cervical hasta o mas allá del himen o con inversión de la cúpula vaginal $>50 \%$ de su longitud y descenso de la pared vaginal anterior hasta o mas allá del himen. Se practicó una aproximación vaginal con suspensión 
sacroespinosa bilateral de la cúpula a 48 mujeres y 40 a mujeres se les practicó una aproximación abdominal con suspensión colposacra y reparo paravaginal. Se realizaron procedimientos auxiliares cuando estaban indicados. Se hicieron exámenes pélvicos cuidadosos en el postoperatorio por parte del coautor no cirujano, cada año hasta 5 años. Las mujeres se examinaron en posición de pie durante un esfuerzo máximo. La cirugía se clasificó como óptimamente efectiva cuando la mujer permanecía asintomática, el apex vaginal estaba sostenido por encima de la placa del elevador y no ocurrió ninguna protrusión de tejido vaginal mas allá del himen. La efectividad quirúrgica se consideró como insatisfactoria si la mujer estaba sintomática, el apex descendía $>50 \%$ de su longitud o la pared vaginal protruía mas allá del himen.

Resultados: Ochenta mujeres (42 del grupo vaginal, 38 del grupo abdominal) estuvieron disponibles para la evaluación entre el año y los 5.5 años (promedio 2.5 años). Los grupos fueron comparables en edad, peso, paridad y estado estrogénico, y $56 \%$ habían tenido una cirugía pélvica anterior. No hubo diferencia significativa entre los grupos en morbilidad, complicaciones, cambio en la hemoglobina, dispareunia, dolor o estancia hospitalaria. El grupo vaginal tuvo mayor duración de uso del catéter, mas infecciones del tracto urinario, mas incontinencia, menor tiempo operatorio y menor costo hospitalario. La efectividad quirúrgica fue óptima en el $29 \%$ del grupo vaginal y $58 \%$ del grupo abdominal y fue insatisfactoria requiriendo reoperación en un 33\% del grupo vaginal y 16\% del grupo abdominal. Las reoperaciones incluyeron procedimientos para incontinencia urinaria recurrente en el 12\% del grupo vaginal y $2 \%$ del grupo abdominal. El RR de efectividad óptima por la vía abdominal es 2.03 (IC 95\% 1.22 a 9.83) y el RR de resultado insatisfactorio usando la vía vaginal es 2.11 (IC $95 \% 0.90$ a 4.94 ).

Conclusiones: La cirugía pélvica reconstructiva para la corrección de los defectos significativos del soporte pélvico fue mas efectiva con la aproximación abdominal.

\section{Histerectomía después de la ablación endometrial}

\author{
James B. Unger, MD, and G. Rodney Meeks, MD
}

From the Department of Obstetrics and Gynecology, Marshfield Clinic, and the Department of Obstetrics and Gynecology, University of Mississippi Medical Center

Am J Obstet Gynecol 1996; 175: 1432-1437.

Objetivos: Determinar el número de mujeres a quienes se les practica histerectomía después de la ablación endometrial y las indicaciones para la cirugía subsecuente.

Diseño del estudio: Se practicó ablación endometrial con esfera a 42 mujeres premenopáusicas quienes tenían severa menorragia asociada con un resultado normal del examen clínico, entre noviembre de 1990 y diciembre de 1991. Se evaluaron 37 mujeres a quienes se les ha hecho cuidado continuo revisando la historia clínica. Se entrevistaron por teléfono cuatro mujeres quienes recibieron cuidado en otra localidad. Una mujer se perdió del seguimiento. Las pacientes fueron seguidas por un mínimo de 4 años. Se analizaron la edad, paridad, tiempo operatorio, prepara- ción endometrial, esterilización preablación y dismenorrea preablación con respecto a la histerectomía subsecuente. La satisfacción de la paciente se evaluó a los 24 meses. Se hicieron análisis de tablas de vida para determinar la probabilidad acumulativa de histerectomía.

Resultados: Se practicó histerectomía a catorce de las 41 mujeres (34\%) dentro de los 5 años siguientes a la ablación endometrial con esfera. La hemorragia menstrual anormal continuada y el dolor menstrual se asociaron significativamente con la histerectomía subsecuente. Once de los 14 casos de histerectomía se asociaron con anormalidad macroscópica tales como miomas, adenomiosis, endometriosis y hematosálpinx crónico. Se observó una relación linear entre la histerectomía y el tiempo.

Conclusión: Con base en los hallazgos un tercio de las mujeres a quienes se les practica ablación endometrial con esfera para menorragia pueden esperar que se les practique histerectomía dentro de los 5 años siguientes. Si la relación linear observada durante los primeros cinco años se extrapola, teóricamente, todas las mujeres necesitarán histerectomía alrededor de los 13 años. La mayoría de las pacientes requirieron histerectomía por anormalidades pélvicas significativas. Se requieren más estudios con seguimiento a largo plazo para definir el papel de la ablación endometrial para la menorragia.

\section{El papel de la colpocleisis con alargamiento uretral en la faloplastia transexual}

Ralph R. Chesson, MD, ${ }^{\mathrm{c}}$ David A. Gilbert, MD, ${ }^{\mathrm{a}}$ Gerald H. Jordan, MD, ${ }^{b}$ Steven M. Schlossberg, MD, ${ }^{b}$ Gerald T. Ramsey, $\mathrm{PhD}$, and Deborah M. Gilbert, $\mathrm{RN}^{\mathrm{a}}$

\section{From the Department of Plastic Surgery, ${ }^{a}$ Urology, ${ }^{b}$ and Obstetrics and Gynecology, ${ }^{c}$ The Eastern Virginia Medical School}

\section{Am J Obstet Gynecol 1996; 175: 1443-1450.}

Objetivo: La cirugía transexual es un área única de intervenciones poco frecuentes. Este estudio examina los factores que tienen significancia en la prevención de la morbilidad mayor en esta cirugía inusual. Se presenta el papel del ginecólogo en el manejo psicológico, endocrino y quirúrgico.

Diseño del estudio: Las cirugías iniciales se complicaron con fístulas en el sitio de la anastomosis uretra-falo. Después de revisar estas complicaciones, modificamos la aproximación incluyendo un procedimiento de dos etapas permitiendo la curación antes de la microcirugía y preservando la pared vaginal anterior durante la histerectomía vaginal y colpocleisis. Preservando la pared vaginal anterior, podemos extender mejor la uretra para unión posterior al falo.

Resultados: Realizando el procedimiento en dos etapas en la colpocleisis se permite una reducción significativa en la tasa de fístulas $(\mathrm{p}=0.0087)$. Con la eliminación de las fístulas, es posible el uso de endurecedores durante la faloplastia para mejores resultados funcionales.

Conclusión: La extensión de la uretra durante la colpocleisis permite una curación mejor y disminuye significativamente la formación de fístulas. Un flujo sanguí- 